\title{
CONFIGURACIÓN DE SUBJETIVIDAD EN MUJERES EN SITUACIÓN DE DISCAPACIDAD: UN ABORDAJE DESDE DISCAPACIDAD, CUERPO Y GÉNERO
}

\author{
CONFIGURATION OF SUBJECTIVITY OF WOMEN IN DISABILITY: AN APPROACH \\ FROM DISABILITY, BODY AND GENDER
}

\section{Pamela Gutiérrez M. ${ }^{1}$, Evelyn Tabilo M. ${ }^{2}$, Eliazar Luna G. ${ }^{3}$, Silvana Catoni A. ${ }^{4}$, Francisco Nilo $G^{5}$, Camilo Bartolomé E. ${ }^{6}$.}

\begin{abstract}
Resumen
La presente investigación tiene por objetivo conocer distintas maneras en que mujeres que se encuentran en situación de discapacidad configuran su subjetividad. En la literatura se abarca el tema del cuerpo desde la terapia ocupacional; sin embargo consideramos que es necesario indagar sobre la constitución de la/el sujeta/o de intervención, específicamente aquel identificado socialmente como mujer y discapacitada/o y así aportar nuevas reflexiones dentro de nuestra práctica profesional. El concepto de subjetividad se aborda en esta investigación desde los ejes de género, discapacidad y corporalidad. Tomando en cuenta los diversos significados a abordar, se decidió utilizar producciones narrativas, una metodología cualitativa, con un diseño narrativo de investigación Dicha metodología entiende el conocimiento como una co-construcción entre el equipo y las/los sujetas/os de estudio. La muestra se da a través de informantes claves y bola de nieve. Como resultado del proceso investigativo se articulan experiencias y saberes de las participantes y el equipo investigador lo cual culmina en una producción narrativa de autoría de cada participante y una narrativa colectiva generada por el equipo investigador. La principales conclusiones apuntan a cómo a partir de la vivencia de una situación de discapacidad fue necesario una reestructuración de los discursos y las prácticas de las mujeres participantes orientadas hacia el uso del espacio público.
\end{abstract}

\section{Palabras clave:}

Discapacidad, género, cuerpo, subjetividad, espacio público.

1 Terapeuta Ocupacional, Licenciada en Ciencias de la Ocupación Humana, Doctora en Psicología Social .Profesora Asistente, Universidad de Chile. Avda. Independencia 1027, Independencia, Santiago Chile. 29786545 email de contacto: pamelagutierrez@med.uchile.cl.

2 Terapeuta Ocupacional, Licenciada en Ciencias de la Ocupación Humana. Terapeuta Ocupacional de Centro Diurno del Adulto Mayor, Padre Las Casas, Región de la Araucanía. Código Postal 4850761. +56985982546. Email de contacto eve.tabilo@gmail.com

3 Terapeuta Ocupacional, Licenciado en Ciencias de la Ocupación Humana. +56976514152. Código postal 9251492. Email de contacto: elo. luna.g@gmail.com

4 Terapeuta Ocupacional, Licenciada en Ciencias de la Ocupación Humana. Docente adjunta Escuela de Terapia Ocupacional, Universidad San Sebastián. Lota 2465 Providencia, Santiago Chile. +5693252809. Email de contacto: silvana.catoni@gmail.com

5 Terapeuta Ocupacional, Licenciado en Ciencias de la Ocupación Humana. Jefe de la Unidad de Terapia Ocupacional del Servicio de Rehabilitación Hospital Dr. Augusto Riffart - Castro, Chiloé. Tutor Clínico Universidad Austral de Chile. +56964895439. Email de contacto: francisconilogaray@gmail.com

6 Terapeuta Ocupacional, Licenciado en Ciencias de la Ocupación Humana. Código Postal 8280073. +5491138766171. Email de contacto: camilo.bartolome@gmail.com 


\begin{abstract}
:
This research aims to find ways in which women that are in disability shape their subjectivity. In literature, the theme of body is addressed from occupational therapy; however it is consider necessary to investigate the constitution of an intervention subject that is specifically identified socially as a woman and disabled and, thus, provide new insights into our practice. The concept of subjectivity in this research is approached from the axes of gender, disability and corporeality. Taking into account the various meanings to address, we decided to use narrative productions, a qualitative methodology, with a narrative research design. This methodology understands knowledge as a co-construction between the team and the subject of study. The sample is given by key informants and snowball. As a result of the research process, experiences and knowledge of the participants and the research team culminates in a narrative production authoring each participant and a collective narrative generated by the research team are articulated. The main conclusions point out how from the experience of disability restructuring the discourses and practices of women participants was necessary, oriented to the use of public space.
\end{abstract}

\title{
Keywords:
}

disability, gender, body, subjectivity, public space.

\section{INTRODUCCIÓN}

La vivencia de una situación de discapacidad con impacto corporal, entendida ésta como aquella discapacidad desde el modelo biomédico homologable a la discapacidad física, y su relación con la configuración de la subjetividad en mujeres, implica desde ésta investigación la compleja interrelación de los conceptos de corporalidad, género y discapacidad. Al respecto, Díaz y Muñoz (2005) exploran el concepto de género como una categoría de construcción sociocultural, política e histórica, y su relación con el concepto de discapacidad, con la finalidad de conocer las implicancias que esta relación tiene en la construcción de la identidad y subjetividad de personas en situación de discapacidad, estableciéndose repercusiones que se relacionan con la pérdida de roles, disminución de la participación y sentido de pérdida de capacidades. Asimismo, Brogna (2009, p. 184) describe este proceso en base al modelo social de discapacidad, estableciendo que los procesos anteriormente mencionados se producen en una matriz sociocultural, donde existe un conjunto de roles que la sociedad espera de las personas y que les son despojadas a las personas en situación de discapacidad por ser percibidas como inferiores.

En relación a los significados de experiencia subjetiva de cuerpo que construyen las personas en situación de discapacidad física adquirida, Gálvez, Godoy y Lagos (2010) señalan que:
"La adquisición de una discapacidad actúa como un dispositivo de cambio significativo que fractura en términos simbólicos la construcción de experiencia de cuerpo, lo que nos permite valorar cambios no sólo en la condición natural del cuerpo, sino también visualizar el cambio social, histórico y cultural del sujeto y su entorno a partir de esta experiencia" (Gálvez, et al, 2010, p. 75). La que sin duda impacta como ya se ha señalado en la los diversos roles desempeñados en la vida cotidiana de las personas.

En este sentido, Ferreira señala que "la normalización médica se ha instalado firmemente en los modos de regulación de nuestras relaciones cotidianas: en nuestra experiencia diaria, en nuestras relaciones (con sus expectativas, ilusiones y esperanzas), estamos sometidos a la regulación simbólica de un capital deseable, el del cuerpo sano, bello y bueno" (Ferreira, 2007, p.3). Operando significativamente desde los diversos dispositivos de salud que se despliegan sobre los y las "pacientes" que adquieren una discapacidad con impacto corporal.

En este contexto, las personas en situación de discapacidad, principalmente aquellas que tienen un impacto en su corporalidad, se situarían al otro lado de la norma corporal esperada. Al respecto Ferreira señala que:

"El cuerpo de la discapacidad ha de ser excluido de los circuitos que sustentan la normalización, el disciplinamiento y la dominación simbólica sobre los que se reproducen las actuales economías capitalistas. Sus cuerpos no son susceptibles de las mismas modalidades de domesticación del cuerpo; sus cuerpos atentan con- 
tra los presupuestos de fondo; sus cuerpos evidencian un ser humano que no se ajusta a las directrices impuestas" (Ferreira, 2007, p.9). Es justamente este cuerpo que no se ajusta, lo que subvierte la norma social y es la situación de discapacidad física adquirida con impacto corporal un evento transversal en la biografía de las personas que expresa de manera sólida el cambio de la experiencia de cuerpo.

Ya que, de alguna manera esta diversidad corpórea altera el orden dado a las formas normalizadas de "ser" en el cuerpo y de "hacerlo" funcionar. De este modo, constatamos cómo una situación de discapacidad adquirida modifica y sitúa al cuerpo en una posición de desventaja frente a los cuerpos considerados como "normales", privándolos del ejercicio de roles y al mismo tiempo marginándolos socialmente.

Es así como, la situación de discapacidad va a repercutir sobre la configuración de la subjetividad e interesa de manera particular conocer las relaciones y tensiones que se producen entre el cuerpo, el ser mujer y el vivir una situación de discapacidad. Como equipo investigador buscamos a través de la perspectiva de los conocimientos situados (Haraway, 1995) generar un conocimiento en torno a la vivencia de la situación de discapacidad el cual sea producto del trabajo en conjunto entre el equipo investigador y las/los sujetas/os de investigación.

A partir de estas reflexiones la pregunta que guía esta investigación se refiere a ¿de qué maneras se configura la subjetividad de mujeres en situación de discapacidad física adquirida?.

Por lo expuesto anteriormente, el objetivo general de esta investigación es comprender maneras en que se configura la subjetividad de mujeres en situación de discapacidad de la Región Metropolitana, en Santiago de Chile, el año 2013. Por su parte, los objetivos específicos son:

1. Articular discursos y experiencias de situación de discapacidad en torno a la configuración de la subjetividad en mujeres en situación de discapacidad,

2. Articular discursos y experiencias de corporalidad en torno a la configuración de la subjetividad en mujeres en situación de discapacidad,

3. Articular discursos y experiencias de género en torno a la configuración de la subjetividad en mujeres en situación de discapacidad,
4. Relevar discursos y experiencias en torno a tópicos emergentes vinculados al tema de subjetividad en mujeres en situación de discapacidad.

El conocer maneras en que se configura la subjetividad de mujeres en situación discapacidad con impacto corporal nos permite realizar reflexiones que problematizan la práctica actual de nuestra disciplina, la Terapia Ocupacional, especialmente en el campo de la salud física. Además, es relevante mencionar el aporte de esta investigación, ya que 1 de cada 7 mujeres en nuestro país presenta algún tipo de discapacidad siendo la deficiencia física la más preponderante ocupando un 30\% según datos de la última encuesta de Discapacidad de Chile (Gobierno de Chile, 2004). Por último, destacar que la metodología de la investigación -las producciones narrativas- nos permiten articular conocimientos y experiencias, poniendo énfasis en la relevancia de la/el sujeta/o de estudio como generador/a de conocimiento.

A continuación se desarrollará el marco teórico en base a los conceptos: discapacidad, cuerpo, género y subjetividad. Luego se presenta la metodología utilizada y se llevará a cabo la discusión y análisis a partir de las producciones narrativas generadas por el equipo investigador y las mujeres participantes.

\section{Discapacidad}

Más allá de las distintas concepciones que existen sobre la discapacidad, nos interesa rescatar el carácter dinámico e histórico del concepto, cuyas concepciones han hecho surgir distintos modelos conceptuales y clasificaciones tales como: CIDDM-1, CIDDM-2, CIF². Dentro de estos modelos conceptuales, la teorización sociológica de la discapacidad, desarrollada por Oliver (1990), Crewe y Zola (1983) críticos a la teoría de la normalización, proponen entender al sujeto dentro de las relaciones sociales que lo configuran y no como un elemento aislado. El planteamiento conceptualiza la discapacidad transfiriendo su ubicación desde el individuo a su interacción en la sociedad (Brogna, 2009 , p. 16). A su vez, señala que para contrarrestar la situación de opresión producida por este fenómeno, es

CIDDM-1: Primera versión de la Clasificación Internacional de Deficiencias, Discapacidades y Minusvalías de la OMS (1980); CIDDM-2: Clasificación Internacional del funcionamiento y la Discapacidad, OMS (1999); CIF: Clasificación Internacional del Funcionamiento, de la discapacidad y de la salud, OMS (2001). 
necesario trabajar desde las políticas, promoviendo el desarrollo de políticas de inclusión en desmedro de la compensación individual o deficiencia, cuestionando el impacto de las ideologías dominantes en estas concepciones y en las acciones que los individuos aplican en la sociedad, con el fin de transformar las ideas homogeneizadoras que causan la exclusión de las personas con discapacidad. Sin embargo, cabe destacar que este modelo:

"Ha establecido una dicotomía entre deficiencia (impairment) y discapacidad (disability), entre lo fisiológico y lo social: la deficiencia no se ha considerado digna de atención, puesto que se presupone que es el ámbito de competencia colonizado por la ciencia médica, y se ha puesto el énfasis en la discapacidad, en las estructuras sociales erigidas en torno a la deficiencia" (Ferreira, 2010, p. 57).

Lo anterior podría traer como consecuencia, que "no hay un substrato fisiológico incuestionable sobre el que se erigen unas estructuras sociales opresivas, sino que ese propio substrato es el resultado de dinámicas sociales, económicas, políticas y culturales" (Ferreira, 2010, p. 57). Al respecto, en nuestra investigación, es la existencia de éste substrato fisiológico - el cuerpo, que integra la experiencia de opresión, que cobra relevancia en la experiencia de discapacidad adquirida con impacto corporal, por lo que es un concepto que desarrollaremos a continuación.

\section{Cuerpo}

Según señala Butler (1993), el cuerpo es construido como materialidad inteligible en donde existe una indisolubilidad entre materia y significado. Desde esta perspectiva, el cuerpo es una entidad que sedimenta significados sociales. En este sentido, la sociedad deposita una serie de significados simbólicos en los cuerpos en relación al tipo de sociedad en la cual se está inmerso. De acuerdo a lo planteado por Ferreira (2009), la sociedad actual fundamentada en el sistema capitalista "ha creado una triple asociación que anuda a los cuerpos que lo habitan en la condición de sanos, buenos y bellos o, por mejor decir, en las oposiciones sano/ enfermo, bueno/malo, bello/feo" (Ferreira, 2009, p.47). En este sistema de significados asociados al éxito, se propone la existencia de un prototipo de cuerpo legítimo asociado a la norma. En relación a esto, el cuerpo "discapacitado" se encuentra desviado de la norma por lo que automáticamente se le supone una incapacidad para realizar las actividades que los cuerpos normales pueden desempeñar (Ferrante y Ferreira, 2007, p. 5). Al respecto, Cruz (2003), junto a otros autores, señalan, que este mismo fenómeno se replica particularmente en el caso de las mujeres, en donde la sociedad despoja a las mujeres con discapacidad del rol de madres por percibirlas incapaces de ejercerlo (Dehays, Hichins y Vidal, 2014 p. 67).

Por lo tanto, resulta interesante referirnos al cruce de los conceptos de cuerpo, discapacidad y género, el cual a su vez tiene un sustrato corporal. Al respecto, Butler nos señala, en cuanto a la construcción del cuerpo femenino, que: "Ser hembra es un hecho sin significado alguno, pero ser mujer es haberse vuelto una mujer, o sea obligar al cuerpo a conformarse con una idea histórica de "mujer", a inducir al cuerpo a volverse un signo cultural, a materializarse obedeciendo una posibilidad históricamente delimitada, y esto, hacerlo como proyecto corporal sostenido y repetido" (Butler 1988, p. 300), lo que se genera en base a una socialización y habituación del cuerpo al género asignado.

Asimismo, De Barbieri (1997) señala que las mujeres se constituyen socialmente como sujetos de deseo donde el cuerpo toma una posición central. Entendido así, la corporalidad femenina tiene implicancias en la construcción de la subjetividad, de modo que las mujeres pueden concebirse como seres que desean ser deseadas, por lo que si su corporalidad no es la esperada, esa construcción y entendimiento es socialmente cuestionada. Éste es un aspecto que complejiza la experiencia corporal de una situación de discapacidad con impacto corporal y genera un espacio de reconfiguración directamente relacionada con la experiencia de cuerpo y género.

\section{Género}

El concepto de género desde su origen problematizó el sexo como el conjunto de fenómenos del orden de lo corporal mientras que género como los ordenamientos socioculturales diversos y construidos a partir de dichas diferencias culturales (De Barbieri, 1997). Siguiendo esta línea, Simone de Beavouir señala que "la mujer no nace, llega una a serlo" (De Beauvoir en Butler, 1990, p.224) en este sentido, el género no es una identidad estable, es más bien, una identidad débilmente constituida en el tiempo, que se va modelando por los gestos corporales, los movimientos y las normas de todo tipo, que constituyen la ilusión de un yo (Butler, 1997). Por 
lo tanto, género es una construcción social que "transforma la sexualidad biológica en productos de la actividad humana" (Rubin, 1986, p.97).

Destacamos la definición de género de Joan Scott (1986) y que declara dos enunciados relevantes a esta investigación, en el primero hace referencia a lo que se ha desarrollado hasta ahora explicando que el género se constituye como la construcción de las relaciones sociales en base a las diferencias de los sexos, en la segunda declara que "el género es una forma primaria de relaciones significantes de poder" (Scott, 1986, p. 366) lo que ha llevado a una distribución del poder en forma jerárquica en los distintos ámbitos de la vida social y política que en las sociedades occidentales ha sido desventajoso para la mujer, siendo el sistema históricamente heredado el patriarcado. Una de las ideas claves en que se expresa esta desigualdad es desarroIlada por Collin (1994) referente a la ocupación del espacio público, señalando que la estructura de géneros ha generado distintos espacios para hombres y mujeres, llevando al hombre a desarrollar su vida en el espacio público-político y relegando a la mujer al espacio privado-doméstico, infinitamente menos valorado conllevando a la sistemática invisibilización de las mujeres.

Entendiendo esta distribución desigual de poder, sumada a que en nuestra sociedad la situación de discapacidad está asociada a un constante proceso de discriminación y exclusión desde ámbitos simbólicos, económicos y sociales (Ferreira, 2009), entendemos como señala Soler y Teixeira (2008), la existencia de una doble discriminación en las mujeres que adquieren una situación de discapacidad con impacto corporal, debido a las desventajas del sexismo y la discapacidad. En relación a esto Morris (1992) explica que las investigaciones deben abordar la discapacidad desde una perspectiva emancipadora, aprovechando la experiencia del movimiento feminista, de manera de no aportar a la imagen victimizadora de la discapacidad y la experiencia individualizada de tragedia personal, sino como un medio que lleve las demandas de derechos del movimiento de discapacidad. Al respecto Morris señala que:

"El movimiento de discapacidad necesita tomar el principio feminista de "lo personal es político" y darle voz a cada experiencia subjetiva, dando valor a nuestras vidas. Si la investigación en discapacidad es emancipadora puede jugar un rol clave en esto." (Morris, 1992 , p. 164). De modo, de modificar los significados culturales de tragedia personal, e insistir en la responsabilidad colectiva y política que tiene la investigación social en este ámbito, de modo de no ser reproductora de los estereotipos y prejuicios en discapacidad.

Por último, a continuación, se desarrolla el concepto de subjetividad explicando cómo este constituye el nodo donde se articulan y tensionan la experiencia de situación de discapacidad con impacto corporal en mujeres.

\section{Subjetividad}

La subjetividad es entendida por Foucault (1984) como "una forma histórica, sujeta a los discursos y prácticas que una sociedad establece" (Foucault en Amigot, 2007, p. 20), así, la subjetividad se construiría en la relación entre sujetos en un momento histórico. Complementario a esto, Butler señala que en este proceso de construcción de subjetividad ocurre el fenómeno de sujeción, considerado como un "proceso de devenir subordinado al poder, así como el proceso de devenir del sujeto" (Butler, 1997, p. 12), a partir de esto, se comprende la subjetividad como un proceso de construcción continua que se da en una relación de sumisión y agencia respecto de los discursos imperantes en un determinado momento histórico, creando sujetas/os funcionales a esa sociedad particular y enmarcadas/os dentro de los sistemas de saber/poder. Sin embargo, en este proceso existe la posibilidad de cambiar los sistemas simbólicos creando un nuevo discurso, concepto nombrado como capacidad de agencia (Butler, 1990, p.29).

Una de las categorías estrechamente relacionadas al concepto de subjetividad, en cuanto construcción social y ordenamiento sociocultural diverso (De Barbieri, 1997) y cambiante a lo largo del tiempo, es el de género. Según señala Butler, el núcleo de la subjetividad así como la identidad del sujeto, se encuentra atravesada por el género, ya que da pautas de coherencia y continuidad definitorias a la categoría de sujeto. Al respecto, Butler postula que el género es performativo, es decir, no es propio del sujeto, sino que es una construcción social que hace que el sujeto actúe dicho género en sociedad y al ser performativo configura la subjetividad, entendiendo ésta como lo que da sujeción y subordina al sujeto. Desde esta perspectiva serían condiciones que preceden a la/el sujeta/o, ocasionándola/o y subordinándola/o desde fuera (Butler, 1988).

Así, en la interrelación con los discursos y prácticas existentes sobre discapacidad se configuran las/los sujetas/os, de esta manera los significados simbólicos construidos socialmente en torno a la situación de disca- 
pacidad ubican a algunas/os sujetas/os en condiciones de inferioridad sobre otras/os. Según señalan Castillo y Muñoz: "quien padece de algún tipo de deficiencia estructural y/o funcional es considerado como incompetente, inválido, descalificado, disminuido, inhabilitado, insuficiente, carente...: todos estos limitantes de la identidad personal, la interacción social y, por tanto, del desarrollo humano" (Castillo \& Muñoz, p. 163).

Dicha construcción de la discapacidad se experiencia en el cuerpo y entendemos que el cuerpo humano es socialmente configurado a partir de los discursos que lo nombran y las prácticas que performa, configurando así, sus dimensiones subjetivas y experienciales. Al respecto, Ferreira señala, que:

"En esa experiencia subjetiva concreta se configura de manera efectiva la corporalidad que nos constituye como seres humanos (...). Esa experiencia subjetiva es la que hay que rescatar en el caso de las personas con discapacidad: la discapacidad como cuerpo socialmente construido y culturalmente reprimido" (Ferreira, 2010, p. 16).

Así, el género, la discapacidad y el cuerpo son constitutivos de la categoría de subjetividad, confluyendo prácticas, discursos, experiencias históricas y dialécticas en la construcción constante de los y las sujeto/ as, que en relación a este trabajo son mujeres que vivencian la situación de discapacidad adquirida con impacto corporal. A continuación se presentan aspectos epistemológicos y metodológicos de la investigación.

\section{Aspectos epistemológicos}

La primera decisión epistemológica guarda relación con la forma de concebir el conocimiento y su origen. En ese sentido, nuestra posición fue concebirlo como construcción social relacional. Nos ubicaríamos, por lo tanto, dentro un paradigma construccionista, al cuestionar un conocimiento objetivo, al que es posible acceder independientemente de la persona que conoce y establecer una verdad más allá de los determinantes sociales que la influencian (Gutiérrez, 2011 p. 68).

Ahora bien, para no caer en el "reduccionismo socio-discursivo" (Sandoval, 2010, p.34), asumimos la existencia de una realidad con cualidades materiales y simbólicas en constante diálogo que dan paso al siguiente punto de relevancia como orientación epistemológica: los conocimientos situados. Haraway señala que "la alternativa al relativismo son los conocimientos parciales, localizables y críticos, que admiten la posibi- lidad de conexiones Ilamadas solidaridad en la política y conversaciones compartidas en la epistemología. El relativismo es una manera de no estar en ningún sitio mientras se pretende igualmente estar en todas partes. La "igualdad" del posicionamiento es una negación de responsabilidad y de búsqueda crítica. El relativismo es el perfecto espejo gemelo de la totalización en las ideologías de la objetividad. Ambos niegan las apuestas en la localización, en el encarnamiento y en la perspectiva parcial, ambos impiden ver bien. La moraleja es sencilla: solamente la perspectiva parcial promete una visión objetiva" (Haraway 1995, p. 329). Por lo tanto, la intención es articular un conocimiento en coherencia con la propuesta epistemológica, que se señala como una co-construcción, ya que es esta condición la que permite comprender que tanto el conocimiento y las realidades se configuran en el proceso de articulación señalado (Sandoval, 2004, p. 18).

\section{Metodología ${ }^{8}$}

Para responder al objetivo de la investigación, ésta fue abordada desde la metodología cualitativa, diseño narrativo y específicamente mediante la metodología de las producciones narrativas (Balash y Montenegro, 2003). Esta metodología se fundamenta en la propuesta epistemológica de "conocimientos situados" de Donna Haraway, quien plantea que "el conocimiento se producirá mediante la conexión parcial, localizable y encarnada con otras posiciones" (Balash y Montenegro, 2003, p.45). En esta investigación se trabajó por una articulación de conocimiento entre el equipo investigador y las/os sujetas/os de investigación, para esto, se llevó a cabo la construcción de un guión narrativo basado en los objetivos de la investigación. El equipo investigador se reunió con cada una de las participantes y mediante una entrevista se abordaron las distintas temáticas. Cada una de las entrevistas fueron transcritas y posteriormente redactadas por el equipo bajo el formato de textualizaciones, es decir, narraciones en primera

\footnotetext{
8 Los aspectos metodológicos de esta investigación fueron presentados en el IV Encuentro de Investigación Cualitativa del Bio-Bío. Departamento de Cs. Sociales de la Universidad del Bío Bío. Escuela de Trabajo Social y Psicología, en Mayo del 2014, bajo el título de "Producciones narrativas en la configuración de subjetividad en mujeres en situación de discapacidad: un abordaje desde discapacidad, género y cuerpo".
} 
persona y recogiendo la forma en que las participantes desean que sea leída su visión del fenómeno. Estas textualizaciones fueron entregadas a las participantes para que fuesen revisadas y corregidas. Luego de una serie de intervenciones, aclaraciones y correcciones, las participantes explicitan la aceptación de la producción narrativa de su autoría9.

El proceso descrito termina con una producción narrativa del equipo investigador, documento que refleja las reflexiones que emanan durante el proceso investigativo y que responden a los objetivos planteados. Para el desarrollo de esta producción narrativa, se ha realizado un grupo focal por parte del equipo investigador (Mella, 2000, p. 4), el que ha sido posteriormente transcrito. Esta técnica permitió discutir y reflexionar, a grandes rasgos, los resultados obtenidos del proceso, sin embargo, el análisis y reflexión de los mismos es de menor recursividad que las narrativas, debido a la limitante temporal de ésta investigación.

La muestra estuvo compuesta por tres mujeres en situación de discapacidad con impacto corporal que residen en la Región Metropolitana. En cuanto a las consideraciones éticas, todas las participantes reciben y firman un consentimiento informado previo a la realización de las entrevistas. En él se explicita la voluntariedad de la participación, libertad de dejarla en el momento que estime conveniente, confidencialidad de la información y metodología de trabajo. El beneficio explícito está conformado por el trabajo conjunto con el equipo investigador, escrito de autoría de la persona libre de utilizar según sus intereses y resignificar experiencias en torno a los objetivos de la investigación.

Respecto de los facilitadores y/o obtaculizadores de esta metodología, cabe destacar que la metodología de las producciones narrativas, al ser un documento textualizado en primera persona, requiere que los/as sujetos/as de investigación posean habilidades de lecto-escritura, así como una comunicación verbal efectiva. Estas habilidades permiten rescatar de forma más fidedigna el discurso de las participantes, existiendo un menor grado de intervención por parte del equipo. En el caso de dos de las participantes de la investigación, existió dificultad en la comunicación verbal, situación que aumentó el tiempo implicado en la transcripción de la información y con ello la posterior textualización.

9 Cabe destacar que una de las producciones narrativas, por motivos de fuerza mayor, no fue posible hacer la última sesión pero si se obtuvo la aprobación de la participante de su uso para la investigación, en el estado de construcción que se encontraba.
Dicha situación se ve reflejada en el bajo número de citas utilizadas en el artículo de investigación, que dan cuenta de los hallazgos y aspectos que emergieron del proceso y que generan debate debido a la congruencia política a la cual nos adscribimos, es decir, el validar a cada participante y su experiencia con igualdad entre ellas y con el resto de la sociedad.

En este aspecto, la presencialidad del equipo investigador-participante es relevante para comprender la intencionalidad y enriquecer el proceso de construcción de la producción narrativa. A su vez, las tecnologías de comunicación existentes aportaron a la fluidez de información con las participantes en cuanto a tiempos de reunión, envío y recibo de la retroalimentación corespondiente.

\section{Implicancias éticas y políticas}

Es relevante considerar las implicancias éticas y políticas cuando representamos experiencias de otros sin explotar, ni traspasar sus derechos humanos (Cohn y Lyons, 2003, p.41). Esto fue resguardado mediante el consentimiento informado y el trato de las narrativas en el texto de la misma forma que las otras autorías, siendo citadas como textos en sí mismos y no sólo como extractos del trabajo de campo y/o transcripciones. En lo político esta metodología toma distancia a aquellas posiciones más positivistas consistentes en "dar voz" (Balasch y Montenegro, 2003, p.46) a otros, posibilitando que sean las mismas involucradas, en articulación con el equipo, quienes releven los discursos que consideran importantes, generándose una relación de horizontalidad entre participantes y equipo en este continuo diálogo que a su vez genera un espacio políticamente responsable (Balasch y Montenegro, 2003, p.47), pues la metodología tiene la flexibilidad para que las participantes modifiquen su discurso de la manera que estimen necesario.

\section{DISCUSIÓN Y ANÁLISIS}

En base a la revisión de las producciones narrativas de cada una de las participantes y la narrativa del equipo investigador encontramos discursos y experiencias relevantes a la configuración subjetiva entorno a los tópicos abordados. Para organizar la información se han dividido las experiencias en categorías que desa- 
rrollaremos a continuación expuestas a través de citas de las producciones narrativas vinculadas a la literatura pertinente. Las categorías desarrolladas serán las de estereotipos, prejuicios y discapacidad ${ }^{10}$, reestructuración de discurso y prácticas, uso estratégico de posiciones y facilitadores y obstaculizadores de las narrativas.

\section{Estereotipo, prejuicio y discapacidad}

Es posible observar en las narrativas, de manera transversal, una tensión en la construcción de la subjetividad entre la visión de la sociedad hacia una persona en situación de discapacidad y lo que cada persona quiere proyectar. A su vez, estas tensiones hacen emerger estrategias de enfrentamiento, tanto a nivel cultural como a nivel corporal. Al respecto de lo último, en su narrativa Quiroz11 señala que:

"Tener distonía afectaba mi imagen corporal (...) se contraponía al andar bien vestida y ser coqueta (...) cuando andas bien vestida dejas la discapacidad de lado (...) la sociedad dice "pobrecita" y no, no soy pobrecita, tengo ropa bacán, la que quiero, la que yo escojo; trabajo, tengo pareja, hago lo que quiero y me visto como quiero y punto" (Quiroz, 2013, p. 5).

Así, Quiroz (2013) busca desmarcarse del constructo social negativo asociado a la discapacidad. En relación a esto, Paul Hunt (1981), activista británico, señala que los estereotipos en la cultura popular son el principal obstáculo para la emancipación de las persona en situación de discapacidad. En esta misma línea Ferreira (2008) señala:

"La identidad social del discapacitado es construida e impuesta desde el entorno no discapacitado, implica heteronomía y, como consecuencia práctica, exclusión y opresión (...) en cualquier caso, es la sociedad la que define esa identidad, la que cultural y simbólicamente "discapacita" a los discapacitados" (Ferreira, 2008, p.7).

En el caso de Quiroz, observamos que debido a la oferta poco llamativa de los discursos y prácticas aso-

10 Entendido como una concepción previa sobre un grupo social determinado, lo que pretende facilitar la economía del pensamiento homogeneizando estos grupos.

11 Quiroz, traductora ingles-francés-español, abogó por los derechos de las personas con discapacidad en el comando electoral de M. Bachelet, actual deportista paralímpica en paraescalada. (Presentación en acuerdo con la autora de la producción narrativa). ciadas a la discapacidad, ella crear su propia imagen, como ella dice, "su propio estereotipo". Así, existe una apropiación estética del cuerpo que busca desmarcarse de la imagen social de la persona "discapacitada". Además, se refleja la capacidad de agencia; logrando una subversión en relación a la imagen esperada de una mujer en situación de discapacidad, buscando modificar los sistemas simbólicos imperantes, creando nuevos discursos y prácticas, al desmarcarse del prejuicio cultural asociado al plano corporal. Sin embargo, existe una tensión en la situación mencionada, puesto que dicha apropiación estética del cuerpo se produce en la medida que se acerca a los cánones estéticos normalizados, es decir, en sumisión a la regulación simbólica del capital corporal deseable, que impera en nuestra sociedad occidental.

Así, los estereotipos señalados se perpetúan, en parte, debido a las estructuras macrosociales. En relación a esto, Pérez $(2013)^{12}$ hace una crítica entorno al rol que le corresponde al Estado Chileno al señalar que: "la discapacidad pasa por un tema de cultura, de educación e información y los Gobiernos tienen responsabilidad en esto" (Pérez, 2013, p. 3). Frente a esta situación, la estrategia para modificar los prejuicios utilizada por Pérez tiene relación con un cambio cultural, pues para ella, el aspecto cultural es clave, y utiliza su rol comunicacional a modo de herramienta para visibilizar a la comunidad de personas en situación de discapacidad en el país, tal como señala: "mi misión en la radio tiene que ver con esto, porque en Chile no somos tema y la sociedad te puede hacer mucho más discapacitada de lo que una silla de ruedas" (Pérez, 2013, p. 3), poniendo así el énfasis en que la sociedad define la identidad cultural que finalmente discapacita.

Asimismo las participantes critican los servicios encargados de llevar a cabo estas funciones al señalar que “¿el Gobierno con SENADIS ${ }^{13}$ qué es lo que hace?. Somos tres millones de personas con discapacidad pero son 13 millones de personas que no tienen cultura de discapacidad" (Pérez, 2013, p.5). En este sentido, en la Ley se señala que una de "las funciones del Servicio Nacional de la Discapacidad serán las siguientes: (...) f) Realizar acciones de difusión y sensibilización" (Gobierno de Chile, 2010, p. 15). Es decir, el cambio cultu-

\footnotetext{
12 Pérez, 29 años, profesora curso electivo Universidad de Chile y conductora Radial. (Presentación en acuerdo con la autora de la producción narrativa).

13 Servicio Nacional de la Discapacidad, Gobierno de Chile.
} 
ral postulado por Pérez (2013) es una responsabilidad que atañe al Estado Chileno.

\section{Reestructuración de discurso y prácticas}

La situación de discapacidad reestructura el discurso y las prácticas de las personas que lo vivencian. Lo que se refleja en la narrativa de Pérez, quien refiere que fue necesaria una reformulación y reestructuración de su proyecto de vida. Al respecto señala que: "tras dos años del accidente comencé a tomar conciencia de la pérdida de tiempo que había vivido, en el cual me sobre exigía haciendo diferentes actividades que hacían que los días no terminaran" (Pérez, 2013, p. 2).

Desde las experiencias abordadas en la investigación, la situación de discapacidad adquirida, opera como un evento vital que requiere realizar una reestructuración de los discursos y prácticas. De acuerdo a lo señalado por Gálvez, et al (2010):

"La adquisición de una discapacidad actúa como un dispositivo significativo que fractura en términos simbólicos la construcción de experiencia de cuerpo, lo que nos permite valorar cambios no sólo en la condición natural del cuerpo, sino también visualizar el cambio social, histórico y cultural del sujeto y su entorno a partir de esta experiencia" (Gálvez, et al 2010, p. 74). Sin duda, es relevante destacar este aspecto de las producciones narrativas y su consideración en los procesos de reestructuración de proyectos de vida, tanto como para las personas implicadas, sus agrupaciones, como para las disciplinas vinculadas a la intervención psicosocial.

\section{Uso estratégico de posiciones}

A lo largo de las producciones narrativas, las mujeres advierten la relevancia de utilizar espacios que les han sido negados de manera sistemática, tanto por el hecho de ser mujer como por el hecho de encontrarse en una situación de discapacidad. Dentro de esta utilización del espacio, existen diferentes formas; pero con el foco puesto en la reivindicación. Al respecto, Rubio $^{14}$ (2013) señala "Yo estaría de acuerdo con que todos tuviéramos un lugar en todos los ámbitos como todas las personas, si no es mucho soñar estar en el

14 Rubio, dueña de casa, al cuidado y crianza de su sobrino. (Presentación en acuerdo con la autora de la producción narrativa). ámbito de la política." (Rubio, 2013, p. 9). Al respecto, Oliver (1990) señala que desde el modelo social de la discapacidad, es necesario incidir justamente en estos ámbitos para generar cambios dentro de los sistemas culturales, políticos y económicos. La importancia de lo anterior también es referida por Quiroz, al señalar que "la discapacidad no es tema en la sociedad chilena, no hay ninguna visión y considero que la única manera de hacer cambios de verdad es a través de la política" (Quiroz, 2013, p. 9).

Dentro del contexto de la política existen una serie de maneras en las cuales se puede incidir en este ámbito, una de ellas guarda relación con utilizar la situación de discapacidad como un facilitador en el uso espacios políticos. En su producción narrativa, Pérez refiere que:

"Me aprovecho de mi condición para hacer lo que hago porque de otra manera hubiera tenido que estudiar periodismo para hacer el mismo programa radial y aún así no sería lo mismo. En ese contexto es mi obligación visibilizar el tema de la discapacidad para avanzar hacia una sociedad inclusiva, porque si viviéramos en una sociedad de estas características la discapacidad no sería tema" (Pérez, 2013, p.7).

En esta misma línea, Quiroz señala que: "Me nació esta urgencia de tomarme los espacios que me corresponden y para hacerlo la silla ha sido una herramienta para llegar arriba, y eso es jugar con mi discapacidad, usar la discriminación positiva" (Quiroz, 2013, p. 12).

En ambas citas, cobra relevancia el uso del espacio público ya que en el confluyen discursos y prácticas, que configuran la producción social constantemente en disputa y que tiene un rol inherentemente político.

A su vez constatamos la doble discriminación en torno a la mujeres participantes de esta investigación, por un lado existe una discriminación por ser mujeres y por otro, al pertenecer al colectivo de personas en situación de discapacidad, llevando a poner en disputa su calidad como individuo, tal como postula Amorós (2005, p. 102) al señalar que "yo no soy individuo/a, ya que no genero los efectos sociales y políticos precisos y contrastables de tal condición".

En las producciones narrativas, las participantes identifican la necesidad de conquistar espacios de los cuales han sido históricamente despojadas como colectivo femenino y en situación de discapacidad. Esto en función del ejercicio de reconocerse en su derecho de ciudadanas, en la cual reivindican su condición de sujeto de derecho y además logran tensionar los actuales estereotipos y prejuicios construidos a través de pro- 
cesos históricos, logro realizado en el espacio público por medio de discursos y prácticas contrahegemónicos.

De hecho para la modificación de estos discursos y prácticas, las participantes utilizan las posiciones en las que se encuentran, como facilitadores, para esto son consciente de los mecanismos bajo los que operan las concepciones construidas socialmente entorno a la discapacidad y los efectos que provocan en su entorno social. Cabe destacar la paradoja que constituye esta acción al utilizar el constructo social desfavorable para lograr un beneficio, pero más profundamente porque el sentido de la adopción de la posición estratégica, es la modificación del constructo social desfavorable.

\section{CONCLUSIONES}

A través de la presente investigación, se buscó conocer las maneras en que se configura la subjetividad de mujeres en situación de discapacidad adquirida con impacto corporal. Para lo anterior, abordamos el tema desde cuatro conceptos que consideramos constitutivos de la subjetividad. El primero de ellos, la discapacidad desde la perspectiva del modelo social, matizando esta visión con el concepto de cuerpo como depositario de significados sociales. Por otra parte, el concepto de género se abordó como una relación social, generando relaciones de poder y, por último, nuestro eje central de la investigación, la subjetividad como un proceso dinámico que performa a los y las sujeto/as en torno a los discursos imperantes, permitiendo a la vez el cambio de estos discursos a través de la capacidad de agencia.

En las producciones narrativas, la discapacidad operó como una situación que desencadenó una serie de procesos relacionados con la exclusión, que las participantes tuvieron que enfrentar. En este escenario, su identidad era tensionada constantemente por los discursos imperantes acerca del concepto de discapacidad, y ellas asumieron una postura activista, abogando por el ejercicio pleno de sus derechos en el espacio público.

A su vez, el cuerpo se presenta como materialidad depositaria de significados sociales y de las concepciones de discapacidad en tanto "cuerpo discapacitado", reproduciendo los estereotipos y prejuicios asociados. Sin embargo, el cuerpo puede ser performado en pos de un discurso disidente y problematizador de estos estereotipos y prejuicios, pudiendo llegar a modificarlos o a operar coherentemente hacia la reproducción de los modelos estéticos esperados por medio del consumo de sus símbolos.

Las situaciones descritas anteriormente fueron influenciadas de manera transversal por el género, el cual operó como un obstaculizador en muchas ocasiones del uso del espacio público. Además, el hecho de ser mujer y vivir una situación de discapacidad no implica que estén exentas de la influencia de los discursos imperantes en torno a la femeneidad; sin embargo implicó una experiencia diferente, dado que la sociedad despoja a las mujeres en situación de discapacidad de lo que se espera del género femenino, ya que no se espera un capital corporal bonito, bueno o sano, generando una tensión entre estar o no, sometida a los discursos y prácticas asociados al ser mujer.

Así, a lo largo de la investigación se han hecho visibles cómo, en el caso de estas tres mujeres, han operado estas dimensiones sobre ellas y cómo esto ha tenido un correlato en la construcción de su subjetividad, es decir, la situación de la discapacidad de las mujeres participantes guía el proceso configurativo de la subjetividad, la cual se vuelve aprehensible al performar y narrar en sociedad las distintas ocupaciones en las cuales se involucran.

Dentro de la investigación, nuestros principales hallazgos en torno a la configuración de la subjetividad guardan relación en cómo a partir de este evento fue necesario una reestructuración de los discursos y las prácticas de las mujeres participantes, reestructuración que se va haciendo cuerpo a medida que van encontrando focos que guían el actuar. Este foco muchas veces tuvo relación con el uso del espacio público, ya sea a través de la iglesia en el caso de Rubio, el rol como comunicadora de Pérez y el trabajo político de Quiroz. El uso que se le da al espacio público no es casual, sino que tiene por objetivo visibilizar el tema de la discapacidad y romper con aquellos estereotipos y prejuicios asociados.

Asimismo, se busca a largo plazo generar cambios culturales que den respuesta a estas necesidades, cumpliendo así con lo propuesto en el modelo social de discapacidad, logrando el cuestionamiento de las ideologías hegemónicas, con el fin de generar una sociedad inclusiva para las personas con discapacidad. Estas acciones son necesarias puesto que las personas con discapacidad actualmente se encuentran en una posición de opresión, puesto que, al igual que otros grupos sociales, "siguen siendo sistemáticamente excluidos y dificultosamente pensables en el imaginario de la ciudadanía, donde en definitiva se juega no solo la viabili- 
dad de su representación política, sino, más apremiantemente aún, su superivivencia" (Sabsay, 2006, p. 8).

En cuanto a las limitantes del estudio, como equipo investigador consideramos que las dimensiones abordadas en el estudio no son suficientes para aprehender en profundidad la configuración de subjetividad de las mujeres. Estas dimensiones emergieron a lo largo de las distintas producciones narrativas, donde los factores de nivel educacional, nivel socioeconómico, edad, distribución geográfica, entre otros, pueden incidir de gran forma en la configuración de la subjetividad. Por otra parte, otra limitante del estudio fue el tiempo utilizado en el trabajo de campo, el manejo de las variables: ubicación geográfica, disponibilidad de tiempo de las participantes, la posibilidad no generalizada de recurrir a medios electrónicos para agilizar el ir y venir de la información en la revisión de las producciones narrativas y el restringido tiempo con el que contamos para desarrollar las producciones narrativas que limitó la profundización en el desarrollo de los temas emergentes.

Finalmente, las proyecciones que surgen a partir de este estudio guardan relación con investigar cómo el/la terapeuta ocupacional puede aportar en el uso de los espacios públicos por parte de las personas en situación de discapacidad desde el modelo social de discapacidad; realizar investigaciones similares considerando el género masculino y profundizar acerca de cómo opera la capacidad de agencia de las mujeres en las situaciones descritas a lo largo del estudio.

\section{RefERENCIAS BibLIOGRÁfiCAS}

Amigot, P. (2007). Una tensa oscuridad. Interrogando el abordaje psicosocial de la subjetividad. Psicología y Sociedade 19(3), 20-25. Porto Alegre Sept./Dec

Recuperado de: http://www.scielo.br/pdf/psoc/v19n3/a04v19n3.pdf

Amorós, C. (2005). La gran diferencia y sus pequeñas consecuencias para las luchas de las mujeres. Madrid, Ediciones cátedra.

Balasch, M., \& Montenegro, M. (2003). Una propuesta metodológica desde la epistemología de los conocimientos situados: Las producciones narrativas. Encuentros en Psicología Social, 1 (3), 44-48.

Butler, J. (1988). Actos performativos y constitución del género: Un ensayo sobre la teoría y fenomenología feminista. Theatre Journal, 40 (4). The Johns Hopkins UniversityPress, 519-531. Publicado en español en Debate feminista, 18 (1998): 296-314 Recuperado de: http://www.debatefeminista.com/PDF/Articulos/actosp433.pdf

Butler, J. (1990). El género en disputa. El feminismo y la subversión de la identidad. Barcelona Paidós.
Butler, J. (1993). Bodies that matter: On the discursive limits of "sex". New York: Routledge.

Butler, J. (1997 ). Mecanismos psíquicos del poder. Teorías sobre la sujeción. Stanford, Universidad de Stanford.

Brogna, P. (2009).Visiones y revisiones de la discapacidad. México. Fondo de Cultura Económica.

Castillo, L. Muñoz, P. (2005). Implicaciones del género y la discapacidad en la construcción de identidad y la subjetividad. Revista Ciencia Salud, Universidad del Rosario, Colombia 3 (2), 157 - 167.

Cohn, E.; Lyons, K. (2003). The perils of power in Interpretative Research. American Journal of Occupational Therapy 57, 40-48. doi:10.5014/ajot.57.1.40

Collin, F. (1994). Espacio doméstico, espacio público. En: Ciudad y Mujer. Madrid: Seminario Permanente "Ciudad y Mujer" 231-237. Recuperado de: http://www.webiigg.sociales.uba.ar/grassi/archivos/ Espacio_Domestico.pdf

Cruz, M. (2003). La maternidad de las mujeres con discapacidad física: una mirada a otra realidad 87 - 105. Recuperado de: http://www. debatefeminista.com/PDF/Articulos/lamate958.pdf

De Barbieri, T. (1997). Certezas y malos entendidos sobre la categoría de género. En: L. Guamán y Pacheco, C. (comp.) Estudios Básicos de Derechos Humanos IV. Instituto Interamericano de Derechos Humanos, San José, 47-84.

Dehays, M. et al (2014). Análisis del Significado de las Ocupaciones Atribuidas a Ser Mujer y Madre para un Grupo de Mujeres con Discapacidad Intelectual en la Ciudad de Punta Arenas. Tesis de Grado de Terapeuta Ocupacional. Universidad de Magallanes. Chile. Recuperado de: http://bibliotecadigital.indh.cl/bitstream/ handle/123456789/612/TESIS.pdf?sequence $=5$

Díaz, L. y Muñoz, P. (2005). Implicaciones del género y la discapacidad en la construcción de identidad y la subjetividad. Revista Ciencias de la Salud. Bogotá 3 (2). Recuperado de: http://redalyc.uaemex. $\mathrm{mx} / \mathrm{pdf} / 562 / 56230206 . \mathrm{pdf}$

Ferrante, C. y Ferreira, M. (2007). Cuerpo y habitus: el marco estructural de la experiencia de la discapacidad. Recuperado de: www.um.es/ discatif/PROYECTO_DISCATIF/.../Ferrante_Ferreira.pdf

Ferreira, M. (2007). Una aproximación sociológica a la discapacidad desde el modelo social: apuntes caracteriológicos. Revista Española de Investigaciones Sociológicas (Reis) 124, 141-174.

Ferreira, M. (2008). La construcción social de la discapacidad: habitus, estereotipos y exclusión social. Nómadas. Revista Crítica de Ciencias Sociales y Jurídicas. Universidad Complutense de Madrid. 17 (1). Recuperado de: http://revistas.ucm.es/index.php/NOMA/article/view/27586

Ferreira, M. (2009.) De la minus-valía a la diversidad funcional: un nuevo marco teórico-metodológico. Revista Política y Sociedad. 47 (1). Recuperado de: http://revistas.ucm.es/index.php/POSO/issue/ view/POSO101013/showToc

Ferreira, M. (2010). Discapacidad y corporalidad: una aproximación genealógica. En Brenda A. Bustos (coord.): Cuerpo y Discapacidad: 
perspectivas Latino Americanas, Facultad de Letras - Universidad Nacional de Nuevo León. México.

Gálvez, M., Godoy, M. y Lagos, A. (2010). Construcción de significados de experiencia de cuerpo con personas en situación de discapacidad física adquirida. Revista Chilena de Terapia Ocupacional 10 (71-83). Recuperado de: http://www.revistaterapiaocupacional. uchile.cl/index.php/RTO/article/viewArticle/10562

Gobierno de Chile (2004). ENDISC Primer estudio nacional de la discapacidad en Chile. Recuperado de: http://www.senadis.gob.cl/ documentos/endisc

Gobierno de Chile (2010). LEY 20.422. Establece normas sobre igualdad e inclusión social de personas con discapacidad. Recuperado de: www.leychile.cl/n?=1010903\&f=2011-05-27\&p.

Gutiérrez, P. (2011). Terapia ocupacional: una disciplina para la autonomía. Prácticas y discursos de Gubernamentalidad y subjetivación en torno a la ciencia emergente. Tesis doctoral. Universidad Autónoma de Barcelona. España. Recuperado de: http://hdl.handle. net/10803/285779

Haraway, D. (1995). Ciencia, cyborgs y mujeres. La reinvención de la naturaleza. Madrid. Ediciones Cátedra.

Hunt, P. (1981). Setting accounts with the parasite people: a critique of a life apart. E.J Miller and G.V. Gwynne. Disability Challenge 1: 37-50.

Ibañez, T. (2003). La construcción social del socio construccionismo: retrospectiva y perspectiva. Política y Sociedad. 40.155-160.

Mella, O. (2000). Grupos focales "focus groups" técnica de investigación cualitativa. Publicado como documento de trabajo N³ CIDE. Santiago, Chile. Recuperado de: http://biblioteca.uahurtado.cl/ ujah/856/txtcompleto/txt105091.pdf

Morris, J. (1992). Personal and political: a feminist perspective on researching physical disability. Disability handicap and society, 7 (2) 157-166.
Oliver, M. (1990). The politics of disablement. London: Macmillan.

Pérez, C. (2013). Mi felicidad, una elección desde el autoconocimiento. Producción narrativa. Manuscrito no Publicado.

Quiroz, E. (2013). Saliendo a flote. Producción narrativa. Manuscrito no Publicado.

Rubin, G. (1986). El tráfico de mujeres: notas sobre la "economía política del sexo". Nueva antropología. 8 (30) 52-96.

Rubio, M. (2013). Entre Risas y Lágrimas. Producción narrativa. Manuscrito no Publicado.

Sabsay, L. (2006). La configuración de identidades como posiciones de sujeto: antiesencialismo y diferencia en Judith Butler. Revista internacional de Culturas \& Literaturas, 5. Recuperado de: http://www. escritorasyescrituras.com/revista-detalle.php/5/35/la-configuracinde-identidades-como-posiciones-de-sujeto-antiesencialismo-ydiferencia-en-judith-butler?PHPSESSID=425da30c1a03168d6724 cf15a0f4f73f

Sandoval, J. (2004). Representación, discursividad y acción situada. Introducción crítica a la psicología social del conocimiento. Valparaíso. EDEVAL.

Sandoval, J. (2010) Construccionismo, conocimiento y realidad: una lectura crítica desde la psicología social. Revista Madrid. 23, 31-37.

Scott, J. (1986). Gender: a useful category of historical analysis. American Historical Review, 91, 1053-1075.

Soler, A. y Teixeira, T. (2008). Discapacidad y dependencia: una perspectiva de género. XI Jornadas de Economía Crítica, Bilbao, España. Recuperado de: http://pendientedemigracion.ucm.es/info/ec/ ecocri/cas/Soler_Domingo.pdf

Crewe, N y Zola, I. (1983). Independent living for physically disabled people. San Francisco, Jossey-Bass. 